\title{
Política Nacional de Atenção Básica de 2017: análise da composição das equipes e cobertura nacional da Saúde da Família
}

\author{
National Primary Care Policy 2017: analysis of teams composition \\ and national coverage of Family Health
}

Clarice Brito e Souza Gomes (https://orcid.org/0000-0001-7707-9050) ${ }^{1}$

Adriana Coser Gutiérrez (https://orcid.org/0000-0002-7305-5841) ${ }^{1}$

Daniel Soranz (https://orcid.org/0000-0002-7224-5854) ${ }^{1}$

${ }^{1}$ Escola Nacional de Saúde Pública, Fiocruz. R. Leopoldo Bulhões 1480, Manguinhos. 21041-210 Rio de Janeiro RJ Brasil. britoclarice@yahoo.com.br

\begin{abstract}
The Family Health Strategy is the main form of organization of the Brazilian health system. However, the third edition of the National Primary Health Care Policy (PNAB) recognized other types of teams financially. A time series study was conducted from 2007 to 2019 using data from the National Register of Health Facilities (CNES) of jobs, teams and national coverage of Family Health to analyze the effects of the 2017 National Primary Health Care Policy (PNAB) on team composition. We observed the concentration of doctors in the Southeast and Northeast and variation of this professional category before the events of the "Mais Médicos" (More Doctors) Program. The number of nurses increased 5\% and Community Health Workers (ACS) dropped $0.3 \%$ in the country. Despite the authorization and funding for the implementation of "Primary Care" teams (eAB), they correspond to less than $1 \%$ of the total teams. It is noteworthy that the municipal managers' preferred mode is the traditional Family Health Teams, equivalent to 75\% of the total and growing. While the questionings and expectations generated by the 2017 PNAB in the context of Primary Health Care, we can conclude that, regarding the teams and their compositions, no significant change was identified two years into its coming into force.
\end{abstract}

Key words National primary health care policy, Primary health care, Family health strategy
Resumo A Estratégia Saúde da Família é a principal forma de organização do sistema de saúde brasileiro. Contudo, a terceira edição da Política Nacional de Atenção Básica (PNAB) passou a reconhecer financeiramente outros tipos de equipes. Para analisar os efeitos da PNAB de 2017 na composição das equipes, foi realizado um estudo de série temporal de 2007 a 2019 utilizando dados do Cadastro Nacional de Estabelecimentos de Saúde (CNES) de postos de trabalho e de equipese a cobertura nacional da Saúde da Família. Observou-se a concentração de médicos nas regiões Sudeste e Nordeste e oscilação dessa categoria profissional ante os acontecimentos do Programa Mais Médicos. Houve acréscimo de $5 \%$ de enfermeiros e redução de 0,3\% dos ACS no país. A despeito da autorização e financiamento para implantação de equipes de "Atenção Básica" (eAB), elas correspondem a menos de $1 \%$ do total de equipes. Vale ressaltar que a modalidade preferencial dos gestores municipais se mantem pela Equipes de Saúde da Família, correspondendo a $75 \%$ do total de equipes e em crescimento. Apesar dos questionamentos e expectativas gerados pela PNAB de 2017 no contexto da Atenção Primária à Saúde, conclui-se que, em relação às equipes e suas composições, não houve mudança significativa após dois anos de sua vigência.

Palavras-chave Política nacional de atenção básica, Atenção primária à saúde, Estratégia de saúde da família 


\section{Introdução}

O Programa Saúde da Família (PSF), implantado em 1994 e aprimorado nos anos subsequentes como Estratégia de Saúde da Família (ESF), constitui o principal mecanismo utilizado para induzir a expansão da cobertura da Atenção Primária a Saúde (APS) no Brasil ${ }^{1-3}$.

Até 2006, a APS era regulada por inúmeras portarias e normas publicadas pelo Ministério da Saúde (MS) para apoiar o processo de descentralização do sistema por meio de incentivos financeiros aos municípios e estados brasileiros.

Dentre estes normativos, destaca-se a publicação pelo Ministério da Saúde (MS), em 1996, da Norma Operacional Básica (NOB/SUS/96) ${ }^{4}$, iniciativa determinante para a implantação das equipes do PSF, que modificava completamente a lógica de financiamento e permitia a maior mudança do modelo assistencial vista até então ${ }^{5}$. Assim, com a implantação do Piso de Atenção Básica (PAB fixo e dos incentivos), o PSF passou a ter orçamento próprio de modo a viabilizar sua expansão no país ${ }^{6}$.

Em 2006, o governo federal publicou a primeira Política Nacional de Atenção Básica (PNAB) com o objetivo de estabelecer diretrizes organizacionais, tomando em conta os princípios propostos pelos Pactos pela Vida, em Defesa do SUS e de Gestão e a expansão nacional da ESF, ratificando este modelo como prioritário na condução da APS? .

Construída mediante a utilização de sólida base científica, a PNAB de 2006 deu início à importante discussão da organicidade institucional do sistema em redes de atenção, ao trazer recomendações norteadoras para os serviços de saúde, para o processo de trabalho, para a composição das equipes, para as atribuições das categorias profissionais e para o financiamento do sistema, dentre outros 8 .

Além disso, o seu texto incorporou e difundiu no país os atributos da APS definidos por Starfield ${ }^{9}$, tais como: primeiro contato; longitudinalidade; integralidade; coordenação; orientação para a comunidade; centralidade na família e competência cultural.

Desde sua implantação, muitos estudos demostraram, de diversas formas, os benefícios da Saúde da Família à população brasileira ${ }^{10-23}$, corroborando a literatura internacional, que já apontava em 1920 este caminho aos principais sistemas de saúde universais.

Como parte inerente ao processo de formulação de políticas públicas e a partir da necessidade de adequação aos novos serviços, aliada também à demanda do Conselho Nacional de Secretários Municipais de Saúde ${ }^{24}$, a PNAB passou por duas revisões, em 2011 e 2017. Observa-se que as suas três edições ocorreram em governos distintos, caracterizados por contextos socioeconômicos diferentes, além de os vários programas que atravessaram o seu período de vigência terem sido incorporados nas edições subsequentes (Figura 1).

A edição de 2011, embora tenha reafirmado as diretrizes da edição anterior, flexibilizou a carga horária da categoria médica, com a possibilidade de trabalho por 20 ou 30 horas semanais, visando suprir o déficit destes profissionais nas equipes ${ }^{25}$.

Há o reconhecimento de que a ESF para a população ribeirinha e fluvial, a equipe de Consultório na Rua, o Núcleo de Apoio a Saúde da Família (Nasf), o Programa Saúde na Escola (PSE) e a Academia da Saúde foram inclusões importantes desse processo de revisão da política para a ampliação do acesso e o fomento à resolutividade da atenção em saúde ${ }^{26-30}$.

É válido recordar que houve propostas posteriormente à segunda edição da PNAB, tais como o Instrumento de Avaliação da APS (PCATool), o Programa de Melhoria do Acesso e Qualidade (PMAQ-AB), o Requalifica UBS, o Programa de Valorização do Profissional da Atenção Básica (PROVAB) e o Programa Mais Médicos (PMM), com a finalidade de avaliação de serviço, qualificação da estrutura física e do processo de trabalho através de ofertas educacionais, provimento e fixação de profissional, aliado ao apoio institucional do MS ao conjunto dos estados e municí$\operatorname{pios}^{30-33}$. Compreende-se que a somatória destas iniciativas representa o que se denomina 'catalisadores na implementação da PNAB'.

Em 2015 iniciou-se o processo de revisão da nova PNAB, que, como já apontado por Almeida et al..$^{34}$, foi fortemente marcado por disputas técnico-políticas entre o MS e as instâncias representativas de secretarias municipais e estaduais de saúde, período este caracterizado pelo impeachment da então Presidente da República Dilma Rousseff, em maio de 2016, e a consequente alteração na composição dos dirigentes do MS, agregado à acentuação da crise financeira do país e aos significativos cortes orçamentários na saúde, tais como a Emenda Constitucional (EC 95).

No entanto, o campo da saúde coletiva protagonizou, por um lado, uma infinidade de manifestações nas redes sociais em contestação ao modo e à finalidade para a qual a PNAB estava sendo revista. Foi possível observar um posicio- 


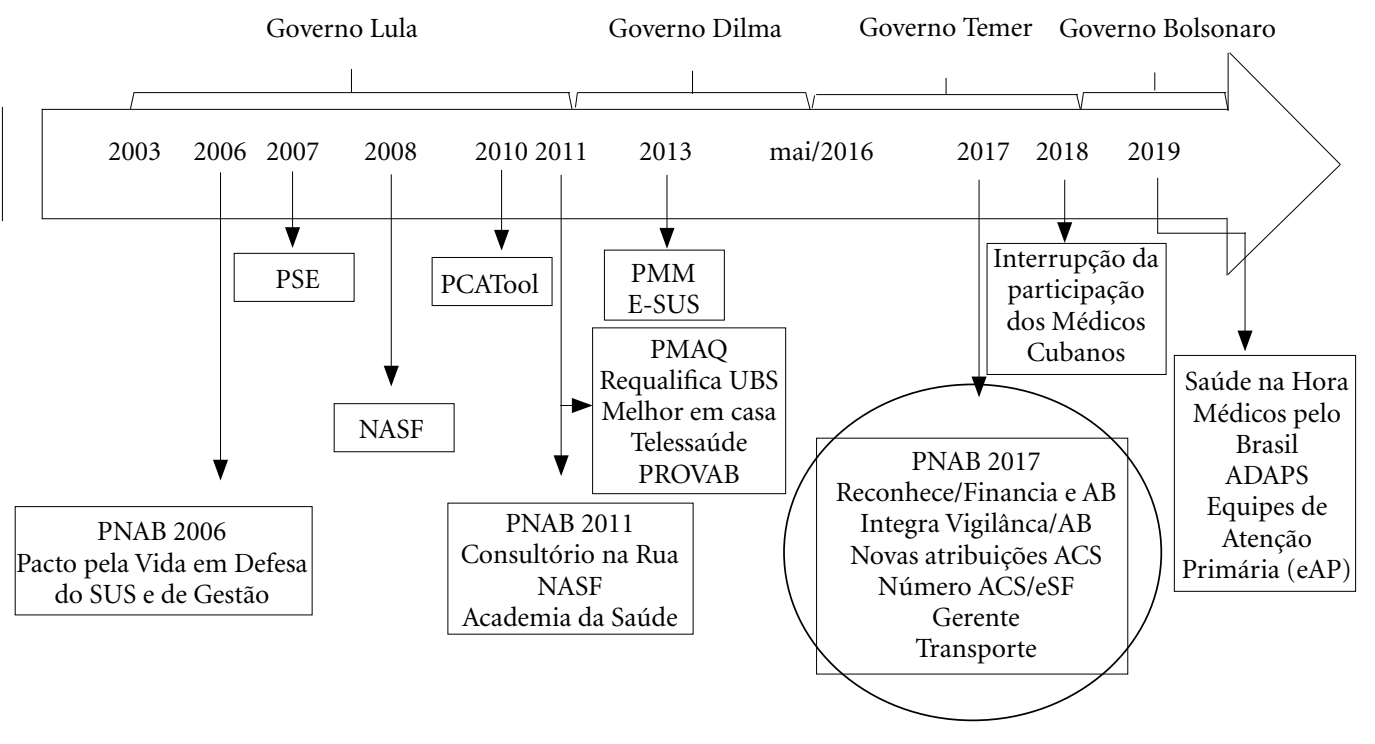

Figura 1. Linha do tempo.

Fonte: elaboração própria.

namento contrário às propostas em virtude da insuficiência do debate mediante a análise de parte destas publicações em sites, tais como: Associação Brasileira de Saúde Coletiva (Abrasco), Centro Brasileiro de Estudos de Saúde (Cebes), Conselho Nacional de Saúde (CNS), Confederação de Agentes Comunitários de Saúde e Agentes de Combates a Endemias (Conacs), Conselho Federal de Enfermagem (Cofen) e Conselho Federal de Medicina (CFM). Por outro lado, o Conass (Conselho Nacional de Secretários de Saúde) e o Conasems (Conselho Nacional de Secretários Municipais de Saúde) reafirmavam a necessidade de reformulação da PNAB para adequá-la à situação de saúde das realidades regionais ${ }^{24,35-39}$.

É provável que os questionamentos à terceira edição da PNAB tenham decorrido também do teor das suas mudanças, as quais vão de encontro às diretrizes vigentes, como o reconhecimento por meio de incentivo financeiro das equipes de Atenção Básica (eAB), o aumento das atribuições dos Agentes Comunitários de Saúde (ACS) e a alteração no seu quantitativo (diminuição) na equipes de SF (eSF), além de sua integração com o ACE (Agente de Combate a Endemias) e de padrões diferenciados para as ações e os serviços de saúde ${ }^{40}$.
Na sequência, a interrupção do convênio com o governo de Cuba, principal parceiro do PMM, gerou preocupação em parte da sociedade no que se refere à permanência do acesso e ao aumento necessário da cobertura da APS $^{41}$. Frente a este cenário e após as eleições presidenciais, o MS divulgou novo edital para a ocupação das vagas.

Vinte e cinco anos após a institucionalização da SF no Brasil, o MS alterou o organograma e criou a Secretaria Nacional de Atenção Primária à Saúde (SAPS), o Departamento de Saúde da Família (DESF) e o novo Departamento de Promoção da Saúde (DAPS) ${ }^{42}$.

Outra mudança em curso foi o Programa Saúde na Hora Brasil, que dá incentivo financeiro à ampliação de horário de funcionamento das unidades de SF, visando ao aumento do acesso nos municípios de médio e grande porte ${ }^{43}$.

Também em 2019 foi publicada a medida Provisória que institui o Programa Médicos pelo Brasil no âmbito da APS, no SUS, e autoriza o Poder Executivo Federal a instituir serviço social autônomo, denominado Agência para o Desenvolvimento da Atenção Primária à Saúde (ADAPS) $)^{44,45}$.

Finalmente, em setembro de 2019, o MS publicou a portaria que extinguiu as $\mathrm{AB}$ e criou as 
equipes de Atenção Primária (eAP), que deverão ser compostas minimamente por médicos, preferencialmente especialistas em Medicina de Família e Comunidade (MFC), e enfermeiros, preferencialmente especialistas em SF, além de vedar a substituição de eSF por eAP, sob pena de suspensão da transferência dos incentivos financeiros ${ }^{46}$.

Embora as recentes propostas venham com a promessa de ampliar o acesso às unidades de $\mathrm{SF}$, bem como de mudar o modelo de financiamento, do provimento e da formação de médicos para áreas remotas, de fortalecer o trabalho em equipe, além da proposição de nova carteira de serviços, a Abrasco ${ }^{47}$ sinaliza para a possível alteração de alguns pilares que vinham favorecendo a estabilidade institucional e o alcance de bons resultados sanitários no país.

Entretanto, diante das sucessivas mudanças mencionadas, resta ao novo governo a discussão dos efeitos da PNAB de 2017 e a necessidade ou não de sua revisão. Fica a pergunta: de fato, o desejo de alguns secretários em criar outros tipos de equipes foi aplicado? E, o quanto esta opção gerou alterações na composição das equipes?

O objetivo deste artigo é apresentar os efeitos práticos da PNAB de 2017 na composição das equipes (eSF,eAB e EACS) e seus efeitos no quadro de profissionais que as compõem.

\section{Métodos}

Trata-se de um estudo de série temporal - 2007 a 2019 -, de abrangência nacional, utilizando dados do Cadastro Nacional de Estabelecimentos de Saúde (CNES) das seguintes variáveis: Recursos Humanos (médicos, enfermeiros e ACS), Rede Assistencial (eSF, EACS eAB) e Cobertura nacional da SF.

$\mathrm{Na}$ análise do CNES/MS, que disponibiliza informações dos "postos de trabalho" a partir da Classificação Brasileira de Ocupações (CBO), utilizou-se dezembro como mês de competência no período de 2007 a 2018 e, no ano de 2019, o mês de agosto, última competência disponível até o fechamento deste artigo.

Realizou-se a tabulação dos dados com auxílio do software Microsoft Office Excel. O cálculo da cobertura de usuários cadastrados considerou a média de 3.450 usuários por eSF implantada, mesma referência utilizada pelo MS, e a população estimada nas projeções anuais do IBGE como base de cálculo para o presente estudo.

Para o cálculo da cobertura cadastrada foram utilizados os dados de cadastro do Sistema de Infor- mação da Atenção Básica (SIAB) e do Sistema de Informação em Saúde para a Atenção Básica (SISAB).

Durante o levantamento dos tipos de equipes no CNES, verificou-se que há diversas classificações e codificações que os subdividem. Como critérios de inclusão, foram selecionadas as equipes que poderiam sofrer prováveis alterações diante das mudanças da PNAB de 2017 e foram excluídas da análise as equipes de Consultório na Rua (eCR), Nasf-AB, eSF transitórias, fluviais (eSFF) e ribeirinhas (eSFR), além de equipes de Atenção Básica do sistema prisional (eABP).

Dessa forma, pretendeu-se relacionar as mudanças ocorridas na última edição da política ao comportamento da APS no país, referente à composição das equipes, na prática assistencial em âmbito nacional.

Em relação ao Sistema de Informação em Saúde, reconhece-se que, apesar do CNES ser a principal fonte de dados oficiais para extrair as informações que o estudo propõe, pode haver inconsistência quanto à alimentação e à atualização das informações na plataforma.

\section{Resultados}

A seguir, os resultados são apresentados de forma a traçar um panorama capaz de contemplar as principais variáveis que caracterizam a composição das equipes de APS, correlacionando-as à Linha do Tempo (Figura 1) apresentada na Introdução.

Na Tabela 1, ao analisar os dados, pode-se salientar a disparidade inter-regional na distribuição dos 'Médicos de Família' e 'Família e Comunidade', em vista de sua maior concentração nas regiões Sudeste e Nordeste do país.

Em 2007, ano base, verificam-se 16.739 cadastros médicos no total Brasil, número que se manteve até 2012. Em 2013, um acréscimo de 4.000 é atribuído provavelmente às mudanças na PNAB de 2011, que flexibilizou a carga horária dessa categoria profissional para enfrentar o seu déficit nas eSF.

Em 2014, observa-se novo acréscimo, o mais importante do período, de quase 7.000, decorrente do Programa Mais Médicos (PMM), instituído em outubro de 2013, elevando o número de cadastros médicos de 'Família e Comunidade' para 27.484 no SUS.

Surpreendentemente, nos anos seguintes, constatam-se incrementos em torno de 1.000 médicos por ano, chegando a 30.181 em 2017, maior número registrado até então na história. 
Tabela 1. Distribuição de Médicos por Região (2007-2019) segundo CBO 2002 - Brasil.

\begin{tabular}{lcccccccccccccc}
\hline & & & \multicolumn{1}{c}{ PNAB } & \multicolumn{4}{c}{ PMM } & \multicolumn{4}{c}{ Saída dos Médicos Cubanos } \\
\hline Região & $\mathbf{2 0 0 7}$ & $\mathbf{2 0 0 8}$ & $\mathbf{2 0 0 9}$ & $\mathbf{2 0 1 0}$ & $\mathbf{2 0 1 1}$ & $\mathbf{2 0 1 2}$ & $\mathbf{2 0 1 3}$ & $\mathbf{2 0 1 4}$ & $\mathbf{2 0 1 5}$ & $\mathbf{2 0 1 6}$ & $\mathbf{2 0 1 7}$ & $\mathbf{2 0 1 8}$ & $\mathbf{2 0 1 9}$ \\
Norte & 1.325 & 1.238 & 1.253 & 1.300 & 1.220 & 1.191 & 1.733 & 2.473 & 2.570 & 2.655 & 2.677 & 2.324 & 2.408 \\
Nordeste & 6.449 & 6.096 & 6.017 & 6.100 & 5.778 & 5.851 & 7.703 & 9.328 & 9.609 & 9.552 & 9.855 & 8.458 & 8.865 \\
Sudeste & 5.604 & 5.533 & 5.645 & 5.895 & 5.882 & 6.193 & 7.105 & 9.589 & 10.004 & 10.471 & 10.871 & 10.035 & 9.646 \\
Sul & 2.304 & 2.120 & 2.157 & 2.227 & 2.255 & 2.312 & 2.897 & 4.258 & 4.403 & 4.400 & 4.655 & 4.227 & 4.286 \\
C. Oeste & 1.057 & 1.023 & 1.031 & 1.118 & 1.075 & 1.228 & 1.337 & 1.836 & 1.896 & 1.941 & 2.123 & 2.073 & 2.087 \\
Total & 16.739 & 16.010 & 16.103 & 16.640 & 16.210 & 16.775 & 20.775 & 27.484 & 28.482 & 29.019 & 30.181 & 27.117 & 27.292 \\
\hline
\end{tabular}

Fonte: Ministério da Saúde - CNES.

Nota(1): mês de competência dezembro (2007-2018) e agosto(2019). Nota(2): médicos (Médico de Família, Médico de família e Comunidade).

Em 2018, com a interrupção da participação de Cuba no PMM, é perceptível a redução de 3.000 vínculos de contratação na Estratégia de Saúde da Família. Quase um ano após a saída do principal parceiro do PMM, ou seja, em agosto de 2019, esse número equivale praticamente ao mesmo valor observado em 2014 .

Ainda em relação à composição das equipes, verifica-se na Tabela 2 a evolução do número de ACS cadastrados no Brasil e a maior concentração dessa força de trabalho na região Nordeste, seguida do Sudeste.

Nota-se em 2007 que os ACS estavam entre as principais categorias profissionais de trabalho atuantes no SUS, chegando a $240.220 \mathrm{mil}$. Em 2012, já haviam crescido em torno de 17\%, chegando a 280 mil, número que praticamente se manteve até agosto de 2019.

Quanto à PNAB de 2017, houve mudanças significativas relacionadas a esses profissionais, que tiveram a sua participação suprimida para, no mínimo, 1 ACS/ eSF.

No entanto, os dados referentes ao período de 2017 a 2019 apresentam redução de 780 agentes comunitários no total Brasil. A análise por região mostra retração de 3\% dos ACS no Sul, $0,4 \%$ no Nordeste e $1 \%$ no Centro-Oeste; já nas regiões Sudeste e Norte houve aumento de $0,8 \%$.

Pode-se comparar ainda na Tabela 2 a evolução de três categorias profissionais que compõem a força de trabalho das eSF. Observa-se discrepância principalmente entre médicos/enfermeiros por equipe. Comparando agosto de $2019 \mathrm{com}$ dezembro de 2017, houve acréscimo de 5\% em relação aos enfermeiros, redução de $10 \%$ dos médicos e $0,3 \%$ de ACS no total do Brasil.

$\mathrm{Na}$ Tabela 2 verifica-se que as equipes de Atenção Básica correspondem a 1\%, quando comparadas ao total de equipes, assim como se nota não ter havido evolução das eAB após a PNAB de 2017.

Observa-se crescimento, em números absolutos, das equipes (eSF, EACS, eAB) no país ao longo do período estudado, porém, entre 2017 e 2019 , nota-se aumento de 2,9\% eSF; 14,2\% EACS e diminuição de $1,7 \%$ de eAB.

Este crecimento se reflete também no aumento da cobertura estimada de Saúde da Família de 1,2\%, mas atenuada pelas estimativas de crescimento da população. Apesar da cobertura estimada ser de 69,9\% em agosto de 2019 , é marcante o contrassenso de somente $42,6 \%$ da população estar cadastrada nos sistemas de informação da Atenção Básica no mesmo período. Além disso, vale ressaltar a interrupção da série histórica diante da mudança do SIAB (Sistema de Informação da Atençao Básica) para o SISAB (Sistema de Informação em Saúde para a Atenção Básica).

\section{Discussão}

Conforme os resultados apresentados neste artigo, que demonstram o expressivo aumento de profissionais dedicados à APS ao longo dos anos, pode-se afirmar, por um lado, que esse é um importante facilitador do acesso e da utilização dos serviços de saúde. Este fator corrobora os achados de Macinko e Mendonça ${ }^{48}$, os quais observaram que a APS no Brasil reduziu a mortalidade infantil e a adulta para algumas condições de saúde sensíveis à atenção primária, melhorou a equidade do acesso e reduziu hospitalizações desnecessárias.

Por outro lado, existem várias barreiras inerentes ao sistema, relativas ao financiamento, à 


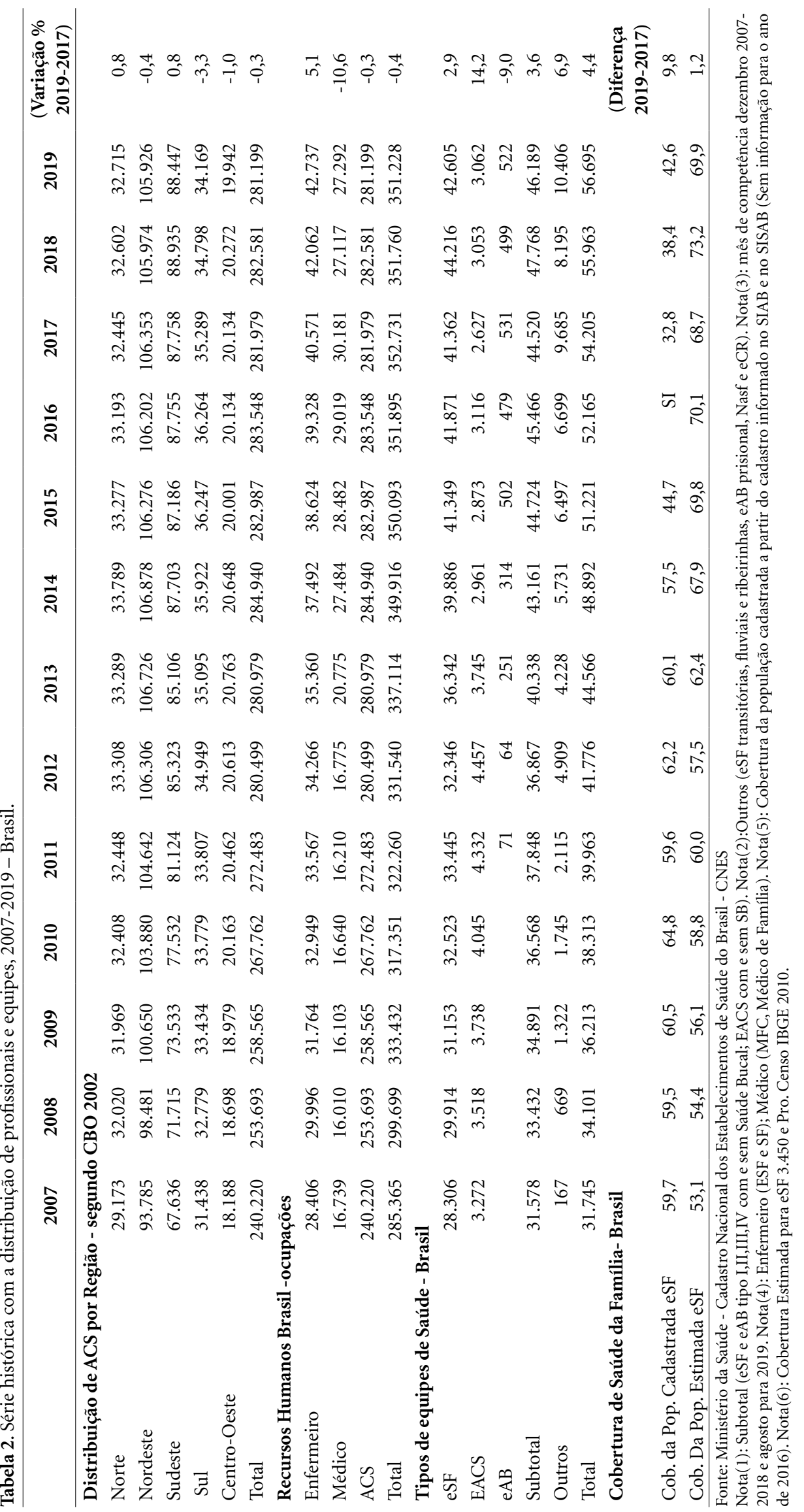


estrutura, à gestão e aos recursos humanos no âmbito do SUS, diante das diversidades dos mais de 5.500 municípios brasileiros, vindo praticamente a estacionar a expansão de cobertura e o número de equipes no País nos últimos anos ${ }^{49}$.

Outro aspecto importante, já mencionado na introdução, refere-se à PNAB adquirir maior relevância organizacional quando acompanhada de programas que se comportam como uma espécie de catalisadores de mudança. Tasca e $\mathrm{Pego}^{50}$ reconhecem que a capacidade do gestor de usar a energia proporcionada pelos programas é determinante para o fortalecimento do sistema de saúde.

No que tange à PNAB de 2017, mesmo com todas as evidências científicas contrárias e ao posicionamento divergente do departamento nacional de atenção básica, a correlação de forças em 2017 favoreceu a implementação de outros modelos diferentes do SF e a redução dos ACS, como relatam Almeida et al. ${ }^{34}$. Entretanto, na prática, verifica-se que a autorização para a implantação de novas eAB não se efetivou (Tabela 2), demostrando que não houve adesão por parte da maioria dos gestores municipais de saúde.

Tal constatação apoia-se também na observação de Cecílio e Reis ${ }^{51}$, de que o reconhecimento (leia-se financiamento) do modelo tradicional de atenção básica é a antiga reivindicação dos gestores dos municípios de médio e grande porte das regiões Sul e Sudeste, as quais contam com baixa cobertura da ESF e resistem à conversão do modelo tradicional de atenção básica para ESF.

Contudo, observa-se na Tabela 2 uma pequena redução no quantitativo de ACS no país, o que, aliada à evolução das eSF no mesmo período, sinaliza para um possível efeito da política estudada, a qual propôs o fim da obrigatoriedade de cobertura dos mesmos para $100 \%$ da população, a supressão de 4 para 1 ACS/eSF, além da não obrigatoriedade desse profissional nas eAB.

Nos últimos dois anos, a mudança mais incisiva observada, que interfere na qualidade e no acesso à saúde, está relacionada ao PMM e não à PNAB. É certo que a dificuldade na provisão de médicos com formação profissional voltada para as necessidades de saúde da população e a sua distribuição no território nacional sempre foi um desafio na trajetória da APS brasileira ${ }^{52}$, situação que permanece até os dias atuais ${ }^{53}$.

$\mathrm{A} \mathrm{OPAS}^{3}$ reconhece o PMM como política pública exitosa, que incidiu na governança do país para os temas de educação e trabalho em Saúde, na ampliação do acesso aos serviços e no processo de transformação da educação, além de ter significado uma retomada estratégica para o enfrentamento do déficit e da distribuição desigual de médicos nos serviços da Atenção Básica no SUS.

Esta iniciativa indutora, entre as duas últimas edições da PNAB, configurou um crescimento exponencial no quantitativo de médicos no país e ampliou a cobertura da SF para municípios mais pobres e de pequeno porte. Contudo, conforme a Tabela 1, no final de 2018, com a interrupção dos cubanos no PMM, houve decréscimo (10\%) comparado a 2017, com potencial ameaça à composição das equipes. Nota-se que, até agosto de 2019, o quantitativo desses profissionais não foi recomposto, mesmo diante das recentes medidas do governo atual visando tal fim.

Em que pese os inúmeros questionamentos e expectativas gerados pela PNAB de 2017 no contexto da APS, pode-se concluir que não se registrou mudança significativa após dois anos de sua publicação em relação às equipes (eSF, EACS, eAB). Houve pequena redução no quantitativo de ACS, que representa menos de $1 \%$ no total Brasil e oscilação no quantitativo de Médicos diante dos últimos acontecimentos do PMM.

Apesar dos importantes avanços de $1994^{1}$ a 2017, quando se analisa a Cobertura Estimada da Saúde da Família no período de 2017 a 2019, verificam-se poucas mudanças, o que pode ser atribuído ao cenário econômico de austeridade ${ }^{54}$, e também à falta de prioridade nos investimentos em saúde e na APS 55 .

Por fim, vale registrar a discrepância entre a Estimativa de Cobertura populacional de SF e a Real Cobertura da população devidamente cadastrada em uma lista atribuída a um profissional ou a uma equipe.

Em 2015, o Ministério da Saúde interrompeu a apresentação das informações públicas de cadastro da população, o que evidencia a descontinuidade dos registros e da organização das listas historicamente alcançados pelo SIAB no seu primeiro manual ${ }^{56}$.

Verifica-se ainda que, no período de vigência do SIAB com os cadastros da "Ficha A", a cobertura estimada e o número de pessoas cadastradas estavam próximas; entretanto, com a criação de outro formato de ficha para a coleta de dados no Sistema de Informação, as informações anteriores se perderam. Tal medida impactou no aumento da discrepância entre as duas formas de cálculo da Cobertura de Saúde da Família. Ou seja, pela série histórica, a Tabela 2 mostra, em 2019, 42,6\% da cobertura da população brasileira cadastrada pelas eSF, enquanto que, em 2010, chegamos a $64,8 \%$. 


\section{Considerações finais}

Em razão do exposto é possível conceber que, apesar de a PNAB ser reconhecida como instrumento legal e relevante na organização da APS brasileira, sua terceira edição, isoladamente, pouco influenciou na composição das equipes e no crescimento de equipes diversas de SF no Brasil.

Tal constatação mostra-se inusitada, ao lembrar a tensão ocorrida no período de reformulação desta política, quando o campo da saúde pública contestou a insuficiência do debate e as propostas expressas em seu texto. Naquela época, as principais instituições representativas do sistema de saúde alertavam, por meio de notas oficiais, para o desmonte do SUS e para as possíveis ameaças às conquistas até então alcançadas no âmbito da APS no país.

Analisando a PNAB de 2017, a partir da definição de política fornecida pelos autores Viana e Baptista $^{57}$, como ação governamental para um setor - o que envolve recursos, atores, arena, ideias e negociação -, conclui-se que a sua implementação não depende exclusivamente das propostas governamentais. Ou seja, considerando que toda política traz intencionalidades, se "o processo ocorrer mediante a negociação entre os entes federativos, os municípios são os protagonistas da execução das políticas" ${ }^{\prime \prime}$.

Deve-se ter em conta, ainda, de um lado, que o reflexo das políticas públicas por vezes demanda tempo e, no caso da PNAB, sua última edição é recente para que se avalie exaustivamente os seus reais efeitos.

Por outro lado, quando se analisam outros programas ministeriais, como o PMM, por exemplo, percebe-se a variação imediata na sua implementação, com o aumento quantitativo no CNES refletindo já no próprio ano de início. Isso comprova que o PMM respondeu a uma demanda real da APS.

Levando-se em conta o acima exposto, ao se comparar os efeitos oriundos da PNAB de 2017 e do PMM, apesar de ambos constituírem inicia- tivas do executivo federal, as referidas políticas públicas mostraram comportamentos bastante diferentes na prática, o que pode ser atribuído ao atendimento ou não das reais necessidades de saúde da população.

Dessa forma, é questionável se as proposições da PNAB de 2017 foram realmente adequadas aos principais problemas públicos que atingiam a APS e se os interesses que motivaram a sua reformulação eram legítimos para a maioria dos municípios, ou se apenas se prestaram à demanda específica de determinada região.

Entende-se que este artigo corrobora a expectativa de Almeida et al. ${ }^{34}$, de que os interesses corporativos, políticos e econômicos possam confluir no sentido da efetivação de uma APS acessível e resolutiva, fortalecendo o SUS como um todo, o que depende substancialmente da participação e do protagonismo da sociedade na luta pelo direito à saúde no Brasil.

Idealmente, espera-se que necessidades sociais e pesquisas científicas embasadas em evidências sejam os principais instrumentos motivadores das políticas públicas em nosso país. Dessa forma, as ações tenderão a promover avanços e resultados que impactem diretamente a saúde das pessoas, honrando os preceitos constitucionais do SUS5.

Para concluir, vale destacar o problema que é calcular a Cobertura de Saúde da Família por estimativa. Enquanto a maioria dos países do mundo e a OCDE utilizam o número de pessoas cadastradas e atribuídas a um médico de família ou a uma eSF (fazendo gestão de cadastros duplicados), no Brasil, desde 1999, insistimos em calcular uma "cobertura potencial" multiplicando o número de equipes por 3.450 pessoas e dividindo-se pela população estimada para o meio do ano pelo IBGE.

Os desafios atuais residem centralmente na vontade política de resgatar os principos de Alma-Ata e os atributos da Atenção Primária, tornando real o acesso e a Cobertura do "Saúde da Família” para toda a população brasileira. 


\section{Colaboradores}

CBS Gomes e D Soranz contribuíram para a concepção, delineamento e análise e interpretação dos dados, redação e tabulação dos microdados, AC Gutiérrez participou da redação e da revisão crítica do artigo.

\section{Referências}

1. Brasil. Ministério da Saúde (MS). Programa de Saúde da Família: saúde dentro de casa. Brasília: Fundação Nacional de Saúde; 1994.

2. Cordeiro H. O PSF como estratégia de mudança do modelo assistencial do SUS. Cad Saúde Família 1996; 1:10-15.

3. Organização Pan-Americana da Saúde (OPAS). Relatório 30 anos de SUS, que SUS para 2030? Brasília: OPAS; 2018.

4. Brasil. Ministério da Saúde (MS). Norma Operacional Básica do Sistema Único de Saúde/NOB-SUS 96. 1997. [acessado 2019 Set 20]. Disponível em: http://www. bvsms.saude/

5. Soranz DR. Reformas de sistemas de saúde informadas em evidências. Cien Saude Colet 2019; 24(6):19941995.

6. Brasil. Ministério da Saúde (MS). Programa Saúde da Família: ampliando a cobertura para consolidar a mudança do modelo de Atenção Básica. Rev. Bras. Saúde Mater. Infant. 2003; 3(1):113-125.

7. Brasil. Ministério da Saúde (MS). Gabinete do Ministro. Portaria no 399/GM, de 22 de fevereiro de 2006. Divulga o Pacto pela Saúde 2006-Consolidação do SUS e aprova as Diretrizes Operacionais do referido Pacto. Diário Oficial da União 2006; 23 fev.

8. Brasil. Ministério da Saúde (MS). Portaria/MS no 648, de 28 de março de 2006. Aprova a Política Nacional de Atenção Básica, estabelecendo a revisão de diretrizes e normas para a organização da Atenção Básica para o Programa Saúde da Família (PSF) e o Programa Agentes Comunitários de Saúde (PACS). Diário Oficial da União 2006; 28 mar.

9. Starfield B. Atenção primária: equilíbrio entre necessidades de saúde, serviços e tecnologia. Brasília: UNESCO, Brasil. Ministério da Saúde ed.; 2002.

10. Rasella D, Aquino R, Barreto ML. Reducing Childhood Mortality From Diarrhea and Lower Respiratory Tract Infections in Brazil. Pediatrics 2010; 126(3):534-540.

11. Giugliani C, Harzheim E, Duncan MS, Duncan BB. Effectiveness of community health workers in Brazil: a systematic review. J Ambul Care Manage 2011; 34(4):326-338.

12. Dourado I, Medina MG, Aquino R. The effect of the Family Health Strategy on usual source of care in Brazil: data from the 2013 National Health Survey (PNS 2013). Int J Equity Health 2016; 15(1):151.

13. Macinko J, Lima Costa MF. Access to, use of and satisfaction with health services among adults enrolled in Brazil's Family Health Strategy: evidence from the 2008 National Household Survey. TM \& IH 2012; 17(1):36-42.

14. Rasella D, Harhay MO, Pamponet ML, Aquino R, Barreto ML. Impact of primary health care on mortality from heart and cerebrovascular diseases in Brazil: a nationwide analysis of longitudinal data. BMJ 2014; 349:g4014.

15. Barreto ML, Rasella D, Machado DB, Aquino R, Lima D, Garcia LP, Boing AC, Santos J, Escalante J, Aquino EM, Travassos C. Monitoring and Evaluating Progress towards Universal Health Coverage in Brazil. PLoS Med 2014; 11(9):e1001692. 
16. Durovni B, Saraceni V, Puppin MS, Tassinari W, Cruz OG, Cavalcante S, Coeli CM, Trajman A. The impact of the Brazilian Family Health Strategy and the conditional cash transfer on tuberculosis treatment outcomes in Rio de Janeiro: an individual-level analysis of secondary data. J Public Health (Oxf) 2017; 40(3):e359-e366.

17. Macinko J, Harris MJ, Rocha MG. Brazil's National Program for Improving Primary Care Access and Quality (PMAQ): Fulfilling the Potential of the World's Largest Payment for Performance System in Primary Care. J Ambul Care Manage 2017; 40(Supl. 2):S4-S11.

18. Santos LPR, Castro ALB, Dutra VGP, Guimarães RM. Internações por condições sensíveis à atenção primária à saúde, 2008-2015: uma análise do impacto da expansão da ESF na cidade do Rio de Janeiro. Cad. Saúde Colet 2018; 26(2):178-218.

19. Andrade MV, Coelho AQ, Xavier Neto M, Carvalho LR, Atun R, Castro MC. Brazil's family health strategy: factors associated with programme uptake and coverage expansion over 15 years (1998-2012). Health Policy and Plan 2018; 33(3):368-380.

20. Soranz D, Pinto LF, Camacho LAB. Analysis of the attributes of primary health care using the electronic medical records in the city of Rio de Janeiro. Cien Saude Colet 2017; 22(3):819-830.

21. Pinto LF, Giovanella L. Do Programa à Estratégia Saúde da Família: expansão do acesso e redução das internações por condições sensíveis à atenção básica (ICSAB). Cien Saude Colet 2018; 23(6):1903-1914.

22. Castro MC, Massuda AD, Almeida G, Menezes-Filho NA, Andrade MV, Noronha KVMS, Rocha R, Macinko J. Brazil's unified health system: the first 30 years and prospects for the future. Lancet 2019; 394(10195):345-356.

23. Gérvas J, Fernándes MP. Organização da Atenção Primária à Saúde. In: Gusso G, Lopes JMC, organizadores. Tratado da Medicina da Família e Comunidade. 2a ed. Porto Alegre: Artmed; 2012.

24. Conselho Nacional de Secretários de Saúde (Conass); Conselho Nacional de Secretarias Municipais de Saúde (Conasems). Nota Conjunta Conasems e Conass sobre a reformulação da PNAB. [acessado $2019 \mathrm{Fev} 20$ ]. Disponível em: http://www.conass.org.br/nota-conjunta-conasms-e-conass-sobre-reformulação-pnab/

25. Brasil. Portaria no 2.488, de 21 de outubro de 2011. Aprova a Política Nacional de Atenção Básica, estabelecendo a revisão de diretrizes e normas para a organização da atenção básica, para a Estratégia Saúde da Família (ESF) e o Programa de Agentes Comunitários de Saúde (PACS). Diário Oficial da União 2011; 29 set.

26. Brasil. Ministério da Saúde (MS). Portaria no 719, de 7 de abril de 2011. Institui o Programa Academia da Saúde no âmbito do Sistema Único de Saúde. Diário Oficial da União 2011; 8 abr.

27. Brasil. Ministério da Saúde (MS). Manual sobre o cuidado à saúde junto a população em situação de rua. Brasília: MS; 2012.
28. Brasil. Portaria GM 154, de 24 de janeiro de 2008. Cria os Núcleos de Apoio à Saúde da Família - NASF. Diário Oficial da União 2008; 25 jan.

29. Brasil. Presidência da República. Decreto no 6.286, de 5 de dezembro de 2007. Institui o Programa Saúde na Escola - PSE, e dá outras providências. Diário Oficial da União 2007; 6 dez.

30. Brasil. Ministério da Saúde (MS). Manual do Instrumento de Avaliação da Atenção Primária à Saúde. Brasília: MS; 2008. (Primary Care Assessment Tool. PCATool-Brasil).

31. Brasil. Ministério da Saúde (MS). Secretaria de Atenção à Saúde. Departamento de Atenção Básica. Programa Nacional de Melhoria do Acesso e da Qualidade da Atenção Básica. Saúde mais perto de você: acesso e qualidade Programa Nacional de Melhoria do Acesso e da Qualidade da Atenção Básica (PMAQ-AB). Brasília: MS; 2012.

32. Brasil. Ministério da Saúde (MS). Requalifica UBS [Internet]. 2014. [acessado 2019 Set 29]. Disponível em: http://dab.saude.gov.br/portaldab/requalifica_ubs

33. Brasil. Lei $n^{\circ} 12.871$, de 22 de outubro de 2013. Institui o Programa Mais Médicos, altera as Leis no 8.745, de 9 de dezembro de 1993, e no 6.932, de 7 de julho de 1981 e dá outras providências. Diário Oficial da União 2013; 20 mar.

34. Almeida ER, Sousa ANA, Brandão CC, Carvalho FFBC, Tavares G, Silva KC. Política Nacional de Atenção Básica no Brasil: uma análise do processo de revisão (2015-2017). Revista Panamericana de Salud Pública 2018; 42:e180.

35. Associação Brasileira de Saúde Coletiva (Abrasco). Nota Oficial Abrasco, 2017. [acessado 2019 Fev 10]. Disponível em: http://www.abrasco.org.br/site/outrasnotiias/notas-oficiais-abrasco/contra-reformulação-da-pnab-nota-sobre-revisão-da-politica-nacional-de-atençao-basica

36. Centro Brasileiro de Estudos de Saúde (CEBES). Nota Cebes: Além de atacar a ESF, ministro debocha da participação popular. 11 ago. 2017. [acessado $2019 \mathrm{Fev}$ 20]. Disponível em: http://www.abrasco.org.br/site/ destaque/nota-cebes-alem-de-atacar-estrategia-desaude-da-familia-ministro-debocha-do-principio-da -participacao-popular

37. Conselho Federal de Enfermagem (Cofen). Cofen reúne-se com agentes comunitários de saúde para tratar da PNAB 2017. 29 set 2017. [acessado 2019 Fev 20]. Disponível em: https://portal.cfm.org. br/index.php?option $=$ com_content $\&$ view $=$ article\&id=27155:2017-09-11-14-25-07\&catid=46:artigos\&Itemid $=18$

38. Conselho Federal de Medicina (CFM). As inconsistências da PNAB. 11 set 2017. [acessado 2019 Fev 20]. Disponível em: https://portal.cfm.org.br/index .php?option $=$ com_content $\&$ view $=$ article $\&$ id $=271$ 55:2017-09-11-14-25-07\&catid=46:artigos\&Itemid $=18$ 
39. Conselho Nacional de Saúde (CNS). Recomendação $\mathrm{n}^{\circ}$ 035, de 11 de agosto de 2017. [acessado $2019 \mathrm{Fev}$ 20]. Disponível em: http://conselho.saude.gov.br/recomendacoes/2017/Reco035.pdf

40. Brasil. Portaria/MS no 2.436. Aprova a Política Nacional de Atenção Básica, estabelecendo a revisão de diretrizes para a organização da Atenção Básica, no âmbito do SUS. Diário Oficial da União 2017; 10 jun.

41. Cuba. Declaração do Ministério da Saúde Pública de Cuba. 14 nov 2018. [acessado 2019 Out 7]. Disponível em: http://www.pt.granma.cu/cuba/2018-11-14/decla ração-do-ministerio-da-saude-publica

42. Reis JG, Harzheim E, Nachif MCA, Freitas JC, D'Ávila O, Hauser L, Martins C, Pedebos LA, Pinto LF. Criação da Secretaria de Atenção Primária à Saúde e suas implicações para o SUS. Cien Saude Colet 2019; 24(9):3457-3462.

43. Brasil. Portaria No 930, de 15 de maio de 2019. Institui o Programa "Saúde na Hora", que dispõe sobre o horário estendido de funcionamento das Unidades de Saúde da Família, altera a Portaria no 2.436/GM/ MS, de 2017, a Portaria de Consolidação no 2/GM/ MS, de 2017, a Portaria de Consolidação no 6/GM/ MS, de 2017, e dá outras providências. Diário Oficial da União 2019; 17 maio.

44. Brasil. Decreto no 9.795, de 17 maio 2019. Aprova a Estrutura Regimental e o Quadro Demonstrativo dos Cargos em Comissão e das Funções de Confiança do Ministério da Saúde, remaneja cargos em comissão e funções de confiança, transforma funções de confiança e substitui cargos em comissão do Grupo-Direção e Assessoramento Superiores - DAS por Funções Comissionadas do Poder Executivo - FCPE. Diário Oficial da União 2019; 21 maio.

45. Brasil. Ministério da Saúde (MS). Portaria GM n ${ }^{\circ}$ 2.539, de 26 setembro de 2019. Diário Oficial da União 2019; 27 set.

46. Brasil. Medida Provisória $n^{\circ} 890$, de 1 ago 2019. Institui o Programa Médicos pelo Brasil, no âmbito da atenção primária à saúde no Sistema Único de Saúde, e autoriza o Poder Executivo federal a instituir serviço social autônomo denominado Agência para o Desenvolvimento da Atenção Primária à Saúde. Diário Oficial da União 2019; 1 ago.

47. Associação Brasileira de Saúde Coletiva (Abrasco). Contribuição dos pesquisadores da Rede APS ao debate sobre as recentes mudanças na política de Atenção Primária, 04 out. 2019. [acessado 2019 Out 7]. Disponível em: //www.abrasco.org.br/site/eventos/ congresso-brasileiro-de-ciencias-sociais-e-humanas/ contribuição-dos-pesquisadores-da-rede-aps-sobre -as-recentes-mudanças-na-politica-de-atençao-primária/43125

48. Macinko J, Mendonça CS. Estratégia Saúde da Família, um forte modelo de Atenção Primária à Saúde que traz resultados. Saúde em Debate 2018; 42(n. esp. $1): 18-37$
49. Santos NR. SUS 30 anos: o início, a caminhada e o rumo. Cien Saude Colet 2018; 23(6):1729-1736.

50. Tasca R, Pego RA. Avaliação de impactos do Programa Mais Médicos: como medir os resultados? Cien Saude Colet 2016; 21(9):2917-2918.

51. Cecilio LCO, Reis AAC. Apontamentos sobre os desafios (ainda) atuais da atenção básica à saúde. Cad Saude Publica 2018; 34(8):e00056917.

52. Machado MH. Os médicos no Brasil: um retrato da realidade. Rio de Janeiro: Fiocruz; 1997.

53. Conselho Federal de Medicina (CFM). Demografia Médica no Brasil. São Paulo: Departamento de Medicina Preventiva da Faculdade de Medicina da USP; Conselho Regional de Medicina do Estado de São Paulo; 2018.

54. Castro MC, Massuda A, Almeida G, Menezes-Filho MA, Andrade MV, Noronha KVMS, Rocha R, Macinko J, Hone T, Tasca R, Giovanella L, Malik AM, Werneck H, Fachini LA, Atun R. Brazil's unified health system: the first 30 years and prospects for the future. Health Policy 2019; 394(10195):345-356.

55. Mendes A, Carnut L, Guerra LDS. Reflexões acerca do financiamento federal da Atenção Básica no Sistema Único de Saúde. Saúde Debate 2018; 42(n. esp. 1):224243.

56. Brasil. Ministério da Saúde (MS). SIAB: manual do sistema de informação de atenção básica. Brasília: MS; 1998.

57. Viana ALD, Baptista TWF. Análise de Políticas de Saúde. In: Giovanella L. Políticas de Saúde e Sistemas no Brasil. $2^{a}$ ed. Rio de Janeiro: Fiocruz, Centro Brasileiro de Estudos da Saúde; 2012. p. 59-88.

58. Domingos CM, Nunes EFPA, Carvalho BG, Mendonça FF. A legislação da atenção básica do Sistema Único de Saúde: uma análise documental. Cad Saude Publica 2016; 32(3):e00181314.

Artigo apresentado em 03/10/2019

Aprovado em 14/11/2019

Versão final apresentada em 16/11/2019 
